carcinogenesis. Although not revealing a statistically significant effect, randomized trials with tamoxifen have aimed at blocking estrogen because male patients with chronic hepatitis present with a relatively hyperestrogenic phenotype (1).

Second, exogenous estrogen therapy by means of oral contraceptives in women is the major risk factor for the development of hepatocellular adenoma (HA) (2), a benign liver tumor with malignant potential. In men, cases of HA after the chronic intake of exogenous steroid hormones have been reported (3).

Third, given that the development of HCC in men with hepatitis $\mathrm{B}$ or $\mathrm{C}$ viral infections or cirrhosis occurs after years of chronic inflammation, we imagine that chronic suppression of IL-6 levels by an estrogen mimetic in those patients would be necessary, and long-term estrogen treatment leads to an even greater risk of HA. In a cohort recently investigated at our institution, 94\% of women with HA reported the use of oral contraceptives for a median duration of 15 years (4). Long-term estrogen treatment in males may also contribute to the development of liver masses. Tumor induction, even if benign, would complicate the clinical management, as any solid tumor in a patient with a history of chronic liver disease should primarily be considered malignant and would be an indication for surgical resection.

DIRK ]. VAN DER WINDT, NIELS F. M. KOK, JAN IJZERMANS

Department of Surgery, Erasmus University Medical Center Rotterdam, Rotterdam 3015 CE, Netherlands.

\section{References}

1. Cancer of the Liver Italian Programme, Lancet 352, 17 (1998).

2. H. A. Edmondson, B. Henderson, B. Benton, N. Engl. J. Med. 294, 470 (1976)

3. E. A. Psatha et al., J. Magn. Reson. Imag. 22, 258 (2005).

4. D. J. van der Windt et al., Br. J. Surg. 93, 1495 (2006).

\section{Response}

THE CONTRIBUTIONS OF SEX STEROID DYSregulation to the development of hepatocellular cancer (HCC) has been debated for decades, spawning several trials studying estrogen modulators (such as tamoxifen) in the treatment of HCC. None of the trials has convincingly shown any benefit of tamoxifen in the treatment of HCC, as Van der Windt and colleagues note. Our study, however, does not address the treatment of existent HCC; it focuses instead on the factors that influence HCC incidence.

We found that estrogen administration markedly diminished the inflammation and injury associated with a chemical carcinogen, which translated much later into a decreased incidence of HCC in mice.

\title{
CORRECTIONS AND CLARIFICATIONS
}

Letters: "Response to 'Shopping for explanations'" by M. M. Krasnow et al. (2 November, p. 745). The first line in the fourth paragraph was incorrect, due to an editorial error. The line should have been, "Finally, only a weak commitment to egalitarianism depends on claims of biological identity."

\section{TECHNICAL COMMENT ABSTRACTS}

\section{Comment on “Roadless Space of the Conterminous United States"}

\section{Evan H. Girvetz, Jochen A. G. Jaeger, James H. Thorne}

Watts et al. (Reports, 4 May 2007, p. 736) introduced a metric of landscape pattern called roadless volume (RV). However, as with most previous metrics, RV does not explicitly address ecological processes. We demonstrate that RV can produce results inconsistent with the notion of landscape connectivity and contend that more ecologically relevant metrics are available.

Full text at www.sciencemag.org/cgi/content/full/318/5855/1240b

\section{Response to Comment on “Roadless Space of the Conterminous United States"}

Raymond D. Watts, Roger W. Compton, John H. McCammon, Carl L. Rich, Stewart M. Wright, Tom Owens, Douglas S. Ouren

Girvetz et al. criticize our work and the value of the roadless volume (RV) metric, basing their arguments on the artificial imposition of wildlife habitat connectivity as the context. We counter that in our intended contexts, both practical and geographic theoretical considerations make RV an appropriate measure of the space between roads.

Full text at www.sciencemag.org/cgi/content/full/318/5854/1240c

Furthermore, when we administered tamoxifen prior to chemical injury, the injury was amplified rather than diminished, suggesting that tamoxifen, already known to be useless in the treatment of established $\mathrm{HCC}$, is also unlikely to prevent the occurrence of $\mathrm{HCC}$.

Although the malignant potential of hepatic adenomas is controversial (1), there is little question that these neoplasms are associated with prior oral contraceptive use in women (2). As Van der Windt et al. point out, the use of estrogen mimetics for prevention of HCC in clinically relevant situations (e.g., chronic liver inflammation such as that produced by hepatitis B or C infection) would be long-term and could lead to the development of adenomas. We agree that this would complicate the care of such patients, likely precluding the use of such a strategy.

Another risk of an estrogen mimetic would be the feminizing side effects in extrahepatic tissues. This includes not only the breasts and testes but also the coagulation system, as estrogens predispose to thrombus formation.

Our results suggest a possible alternative to estrogen-mimetic therapy. The mechanism by which estrogens diminish liver injury is through down-regulation of IL-6 production, indicating that strategies to decrease IL-6 signaling in the setting of chronic liver inflammation may decrease HCC development. Down-modulation of the IL-6 signaling pathway may circumvent the problems of possible adenoma development and feminization inherent in long-term estrogen use, while still providing the benefit of decreasing HCC incidence in men.

MICHAEL KARIN

Laboratory of Gene Regulation and Signal Transduction, Department of Pharmacology and Cancer Center, University of California, San Diego, CA 93093, USA.

\section{References}

1. L. C. Tao, Acta Cytol. 36, 338 (1992).

2. H. A. Edmondson, B. Henderson, B. Benton, N. Engl. J. Med. 294, 470 (1976)

\section{Virtual Reality and Telepresence}

IN THE 24 AUGUST ISSUE, TWO TEAMS OF cognitive neuroscientists used video-based immersive virtual reality systems to induce an "out-of-body" experience in healthy volunteers (Brevia, H. H. Ehrsson, p. 1048; Reports, B. Lenggenhager et al., p. 1096).

An important implication of this work, not fully discussed in the papers, is the role of virtual reality (VR) in inducing these experiences. VR is usually presented as a collection of technological hardware, such as computers and head-mounted displays. However, as underlined by Steuer (1) more than 15 years ago, the core of VR is more experiential than technological; "virtual reality" can be defined as a simulated environment in which a perceiver experiences the feeling of presence by means of a communication medium, a phenomenon referred to as telepresence. 
Using this definition, the outcome of the two studies is immediately clear: During the experiment, the subjects were no longer present in their real body but were instead in the synthetic body produced by VR. In short, the researchers used virtual reality to induce telepresence. But what is "presence," and what is it for?

One group of researchers describes the sense of presence as a function of our experience of a given medium (2). The main result of this approach is the definition of presence as the perceptual illusion of nonmediation (2), produced by means of the disappearance of the medium from the conscious attention of the subject. A second approach (3) based on principles of Embodied Cognition, defines presence as the nonmediated perception of successfully transforming an intention into action.

A better understanding of presence will be an important future goal for cognitive science. This is why the European Community has been funding, since 2002, the "Future and Emerging Technologies-Presence" research program (4).

GIUSEPPE RIVA

Applied Technology for Neuro-Psychology, Istituto Auxologico Italiano, Milan 20145, Italy.

\section{References}

1. ]. S. Steuer, J. Commun. 42, 73 (1992).

2. M. Lombard, T. Ditton, J. Comput. Mediated Commun. 3 (1997); available at http://jcmc.indiana.edu/vol3/issue2/ lombard.html.

3. G. Riva, M. T. Anguera, B. K. Wiederhold, F. Mantovani, From Communication to Presence: Cognition, Emotion and Culture Towards the Ultimate Communicative Experience, G. Riva, F. Davide, Eds. (IOS Press, Amsterdam, 2006); available at www.emergingcommunication.com/volume8.html.

4. Information Society Technologies (www.cordis.lu/ist/ fet/pr.htm).

\section{Response}

WE WELCOME RIVA'S DISCUSSION OF PRESence, virtual reality (VR), and their relevance with respect to our experimental findings on bodily self-consciousness. We also recognize that there is currently no agreement on how to conceptualize and how to measure presence and related aspects such as bodily self-consciousness, whether in the field of engineering, computer science, psychology, or physiology.

It seems to us that Riva overinterprets our data to a certain extent. Unlike Ehrsson (Brevia, 24 August, p. 1048), we did not report that we induced full-blown out-ofbody experiences. True, our participants identified with the virtual body and also experienced touch on the virtual body. They also mislocalized their own bodily selves as they drifted toward their virtual bodies and

\section{Letters to the Editor}

Letters ( 300 words) discuss material published in Science in the previous 3 months or issues of general interest. They can be submitted through the Web (www.submit2science.org) or by regular mail (1200 New York Ave., NW, Washington, DC 20005, USA). Letters are not acknowledged upon receipt, nor are authors generally consulted before publication. Whether published in full or in part, letters are subject to editing for clarity and space.

thus to a position outside their bodily borders. Yet, as we argued in our Report (24 August, p. 1096), this should not be mistaken for full-blown experiences of disembodiment that are typically reported during spontaneous out-of-body experiences. The responses in Ehrsson's study differed from ours: The participants did not feel present in the virtual body, as argued by Riva, but felt located at a distance from their body. Thus, drawing parallels between the two studies and linking them to presence, VR, and bodily self-consciousness is perhaps not as simple as Riva states. Nevertheless, both studies show that experimental paradigms involving multisensory processing in combination with VR might turn out to be an important tool for studying bodily self-consciousness and presence. Experimental psychology and cognitive neuroscience will certainly help to define presence and distinguish between different theoretical accounts.

With respect to one such theory cited by Riva, our data demonstrate that presence (as quantified in our study by the measures of self-location and self-attribution) is not necessarily related to the "perception of successfully transforming an intention in action." We did not modulate motor or intentional mechanisms experimentally and only modified multisensory bodily information. When investigating complex phenomena, such as human bodily self-consciousness or presence, we feel that it is methodologically important to isolate the simplest form of the target phenomenon (in our case, phenomenal selfhood).

We agree that our participants felt some aspects of out-of-body experiences (1). Tackling issues of bodily self-consciousness and presence with different behavioral and neuroimaging techniques is timely, and we expect cognitive science and neuroscience to play a major part in this endeavor.

BIGNA LENGGENHAGER, ${ }^{1}$ TE] TADI, ${ }^{1}$ THOMAS METZINGER, ${ }^{2,3}$ OLAF BLANKE ${ }^{1,4}$
${ }^{1}$ Laboratory of Cognitive Neuroscience, Ecole Polytechnique Fédérale de Lausanne, Swiss Federal Institute of Technology, 1015 Lausanne, Switzerland. 2Philosophical Seminar, Johannes Gutenberg-Universität Mainz, 55099 Mainz, Germany. ${ }^{3}$ Frankfurt Institute for Advanced Studies, Johann Wolfgang Goethe University, 60438 Frankfurt am Main, Germany. ${ }^{4}$ Department of Neurology, University Hospital, 1214 Geneva, Switzerland.

\section{Reference}

1. G. Miller, Science 317, 1020 (2007).

\section{Response}

I DO NOT SHARE RIVA'S IDEA THAT THE concept of telepresence, as formulated in his Letter, will become a useful theoretical framework in cognitive neuroscience. Presence research has been restricted to technologically oriented research departments, and the term has not been used in neuroscience (1). The concept of presence is a "feeling of being there" (in the simulated environment), which leads to the participant responding realistically to events and situations in the virtual environment (1). However, there are many other broader definitions ranging over many possible media - from reading a book or using a mobile phone, to full immersive virtual reality. Steuer (2) described how any technologically mediated communication involves a degree of telepresence, including speaking to someone on the phone, listening to a live music recording, or playing a videogame. For cognitive neuroscientists, this looser definition - together with the fact that this approach to presence relates to many different technological, perceptual, and cognitive factors-becomes a serious limitation. Therefore, I think that it is unlikely that cognitive neuroscientists would find this type of vague definition of telepresence to be very useful in the design and interpretation of experiments investigating specific perceptual and cognitive processes.

Indeed, presence research would benefit from working more closely with cognitive neuroscience to relate presence to psychological or physiological models of perception, action, spatial awareness, body self-representation, and social interaction (3). This could lead to the engineering of the next-generation virtual reality applications in which the simulated sensory data fully replace the input from physical reality, and the feeling of being, acting, and interacting "as if you were there" becomes complete.

\section{H. HENRIK EHRSSON}

The Wellcome Trust Center for Neuroimaging, University College London, London WC1N3BG, UK.

\section{References}

1. M. V. Sanchez-Vives, M. Slater, Nat. Rev. Neurosci. 6, 332 (2005).

2. ]. Steuer, ]. Commun. 42, 73 (1992).

3. M. Slater et al., IEEE Comput. Graph. Appl. 27, 90 (2007). 